\title{
Orientation Correction for Chest Images
}

\author{
Ewa Pietka and H.K. Huang
}

This report presents an automatic procedure that determines the orientation of computed radiography (CR) chest images and rotates them to a standard position to be viewed by radiologists. As an input, CR images of a normalized size of $1,000 \times 1,000$ or $2,000 \times$ 2,000 pixels are used. The analysis is performed in three steps. First, the orientation of the spine within the image is determined. Then, a function searches for upper extremities and the subdiaphragm. Finally, the lungs are extracted and their areas are compared. This indicates whether the image needs to be $y$-axis flipped. These three steps set the value of three parameters on the basis of which the final rotation angle is determined. The procedure has been implemented in the clinics at UCLA. The rate of correctly rotated images is $95.4 \%$.

KEY WORDS: image preprocessing, image orientation, chest radiography, computed radiography, picture archiving and communication system.

$\mathbf{P}$ CTURE archiving and communications systems (PACS) are responsible ${ }^{1-3}$ for acquiring images from several modalities, transfering them to a cluster controller computer for archiving, and distributing them to display workstations. At a workstation the images have to be displayed in a position that is standard. Orientation is one of the problems that has to be addressed before a workstation can be successfully implemented in clinics. The goal of this study is to recognize the orientation of the input chest image and rotate it (if necessary) to a standard position accepted in radiology.

For chest radiographs digitized with a film scanner, the orientation of the image is not a major problem. While digitizing a film, the technologists orient the image to a proper position. However, for computed radiography (CR), the orientation becomes a major problem because of the patient condition and the clinical environment. The CR cassette can be placed at various orientations to accommodate the examination condition. A survey in pediatric radiology found that between $35 \%$ and $40 \%$ of procedures were not performed with the conventional image orientation. Therefore, it is necessary to develop a computerized algorithm to orient these images before they are displayed. This report describes this algorithm, its implementation, and its performance. The procedure is incorporated in an image-processing package ${ }^{4}$ that is working in clinics. Orientation functions have also been developed for abdominal imag$\mathrm{es}^{4}$ and hand images. ${ }^{5}$

The algorithm handles four types of CR chest images: adult posterioanterior (PA) or anteroposterior (AP) and lateral projections and pediatric $\mathrm{PA}$ or $\mathrm{AP}$ and lateral projections. Although all these are chest images, there are different problems that have to be addressed in each of these four types. The adult chest images are much closer to a standard image than the pediatric images in the sense that the mediastinum is usually centered within the image and the image itself contains only the thorax. The problem becomes much more difficult for pediatric images. Their range of variety is much wider, the image may or may not include the head or its part, the location of arms is random, and the area of exposed subdiaphragm differs from one image to another. Very often even the abdominal region is included, changing the location of the lungs within the image. The image is usually not centered and is at a random angle to the vertical axis of the image.

All of these images are analyzed by one algorithm that can detect the wide variety of chest locations within an image. The entire analysis includes two phases. First, the initial analysis reformats the images to a standard $1,000 \times 1,000$-pixel $(1 \mathrm{~K} \times 1 \mathrm{~K})$ or $2,000 \times 2,000$ pixel $(2 \mathrm{~K} \times 2 \mathrm{~K})$ size and removes the unexposed background. Next, the orientation procedure is applied to determine the orientation and rotate the image (if necessary) to a standard position.

From the Department of Radiological Sciences, University of California, Los Angeles, $C A$.

Supported by US Public Health Service grants R01 CA 39063 and P01 CA 51198, awarded by the National Cancer Institute, Department of Health and Human Services.

Address reprint requests to Ewa Pietka, PhD, Department of Radiological Sciences, University of California, Los Angeles, 10833 Le Conte Ave, Los Angeles, CA 90024-1721.

Reprinted with permission from Medical Imaging VI: Picture Archiving and Communications Systems, Society of PhotoOptical Instrumentation Engineers, 1992.

0897-1889/92/0503-0001\$03.00/0 


\section{INITIAL ANALYSIS}

Before the images are subjected to the orientation algorithm, two initial preprocessing steps must be applied. ${ }^{4}$ The first step is to reformat images of various sizes to a standard size. If the size of an input image is larger than $2 \mathrm{~K} \times 2 \mathrm{~K}$, the image is first shrunk in both directions using a two-dimensional bilinear interpolation at a 5:4 ratio. For example, an image of 2,510 $\times 2,000$ size will be reformatted to a size of $2,008 \times$ 1,600 . Second, a suitable number of blank lines is added to extend the size to $2,048 \times 2,048$. If a $1 \mathrm{~K} \times 1 \mathrm{~K}$ image is desired, a subsampling of a $2: 1$ ratio is performed.

For imaging plates that produce pixel matrix sizes smaller than $2 \mathrm{~K} \times 2 \mathrm{~K}$, the image is extended to $2 \mathrm{~K} \times 2 \mathrm{~K}$ by adding blank lines and then subsampled (if necessary) to $1 \mathrm{~K} \times 1 \mathrm{~K}$. The approach is the same whether the original has 8 or 10-bit pixel depth.

The second step is a border-removal procedure. ${ }^{6}$ It removes the unexposed background caused by the blocking of the $\mathrm{x}$-ray collimator and resulting in significant white borders surrounding the image. Border removal reduces the amount of background light in the image in the soft-copy display and minimizes some potential problems that the borders would cause in further image-processing steps. This function is of particular importance in pediatric radiography. In radiographs of infants, the border may cover more than $30 \%$ of the image area. Its removal becomes a necessary stage in the orientation procedure.

\section{ORIENTATION PROCEDURE}

After the unexposed background has been removed, an algorithm is applied to determine the orientation of the image. An image can be oriented correctly or rotated $90^{\circ}$ clockwise, $90^{\circ}$ counterclockwise, or $180^{\circ}$. In each of these cases the image can also be $y$-axis flipped, giving eight possible image orientations. There is no additional information about the type of image (adult or pediatric, PA or lateral view). All these cases are handled by the same algorithm.

The analysis is performed in three steps. First, the direction of the spine is found. This sets a parameter that describes the spine location to 0 for the A, C, E, and G orientations (Fig 1) and 1 for the B, D, F, and $\mathrm{H}$ orientations (Fig 1). The subdiaphragm or the upper extremities are then located. The parameter, describing the location of the upper extremities, is set to 0 for $A$ and $E$, to 1 for $B$ and $F$, to 2 for $C$ and $G$, and to 3 for $\mathrm{D}$ and $\mathrm{H}$ (Fig 1). Finally, the lungs are tested to decide whether the orientation is correct or whether the image has to be $y$-axis flipped. The parameter is set to 0 if no $y$-axis flipping is necessary (Fig 1A-D) or to 1 if flipping is necessary (Fig 1E-H).

In the first step, the average densities are

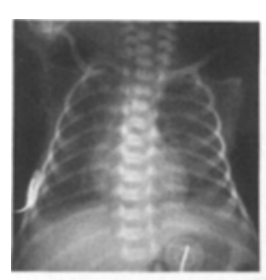

(A)
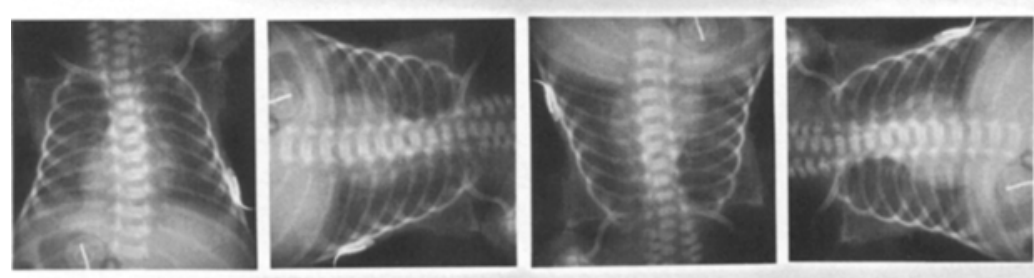

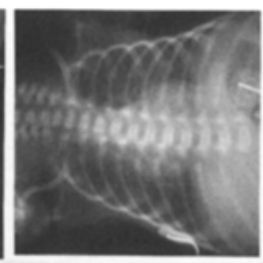

D

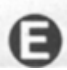

(

G

Fig 1. Eight possible orientations of a chest image. 
calculated along horizontal and vertical lines and are distributed evenly every 100 lines for $1 \mathrm{~K} \times 1 \mathrm{~K}$ images and every 200 lines for $2 \mathrm{~K} \times$ $2 \mathrm{~K}$ images. The average densities are placed in two tables, a horizontal profile table and a vertical profile table. These two profile tables are searched for local maxima whose values and locations within each table are compared. For profiles parallel to the spine direction, the maximum is located close to the middle axis of the image, and its value is larger than the corresponding value of the non-spine-direction profile (Fig 2). In the perpendicular profiles, the maxima are usually in the periphery. The derivative of the profile is also analyzed to test the smoothness (the spine-direction profiles are usually smoother than the non-spine-direction profiles).

Before a decision is made as to which of the two maximum values mark the spine, a doublecheck routine is performed. It searches for the densest area that may belong either to the abdomen or the head (this part has been implemented to eliminate some ambiguity in pediatric cases). This area is marked by two consecutive non-zero-value profiles located at the beginning or end of either profile table that have the largest average value. As a result, the orientation of the spine (horizontal or vertical) is determined, eliminating four orientations.

The second step searches for the neck or upper extremities. It is performed on a thresholded image and is used to find the orientation along the axis perpendicular to the spine. An average of the gray-scale values is calculated over the entire image (after removing the unexposed background) and is used as the threshold value. The thresholding routine marks the contour of the patient and separates it from the patient background (area that is exposed but is outside the patient). The location of the contour line is found by scanning profiles, evenly distributed through the image and perpendicular to the spine (Fig 3). The profiles are then searched for two points (marked by $x$ 's in Fig 3 ) that are the extremities of the longest high-level plateau and are located to the left and right of the mediastinum. Narrow bars, which reflect the presence of intraoperative tubes or background noise, are ignored. The $x$ coordinates of the points create two sequences (one on each
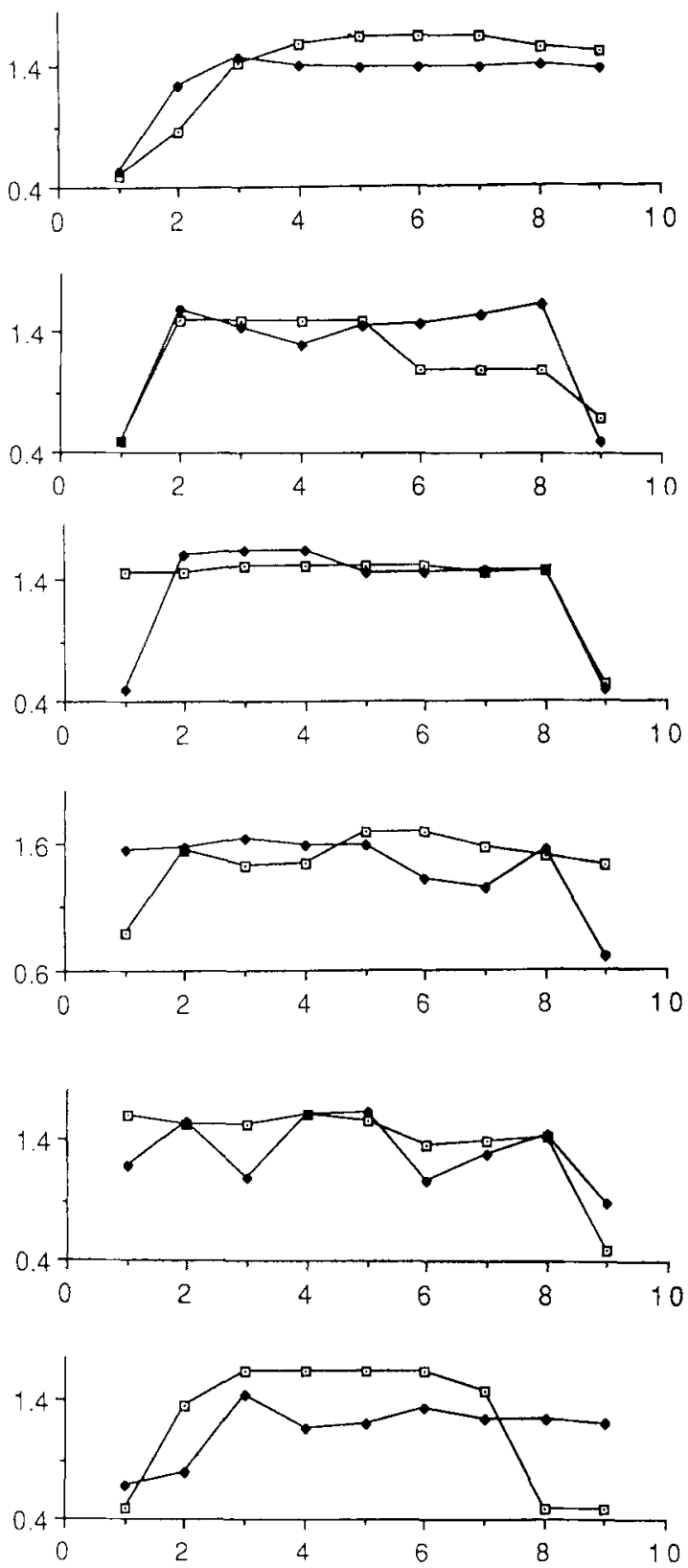

Fig 2. Examples of profiles used for the spine-location analysis. Each plot shows the horizontal and vertical profiles. One of them refers to the spine-direction profile $(\square$; parallel to the mediastinum), and the other is perpendicular to the mediastinum (-). The horizontal axis presents the profile numbers (1-9), and the vertical axis is a normalized average of pixel values computed along lines in the horizontal and vertical directions. The values stored in the table are 1,000 times larger.

side of the mediastinum). Excluding points that belong to the upper-extremity regions, these sequences should be monotonous. If not, the profiles will be incorrectly analyzed and considered again, and the contour point coordinate 

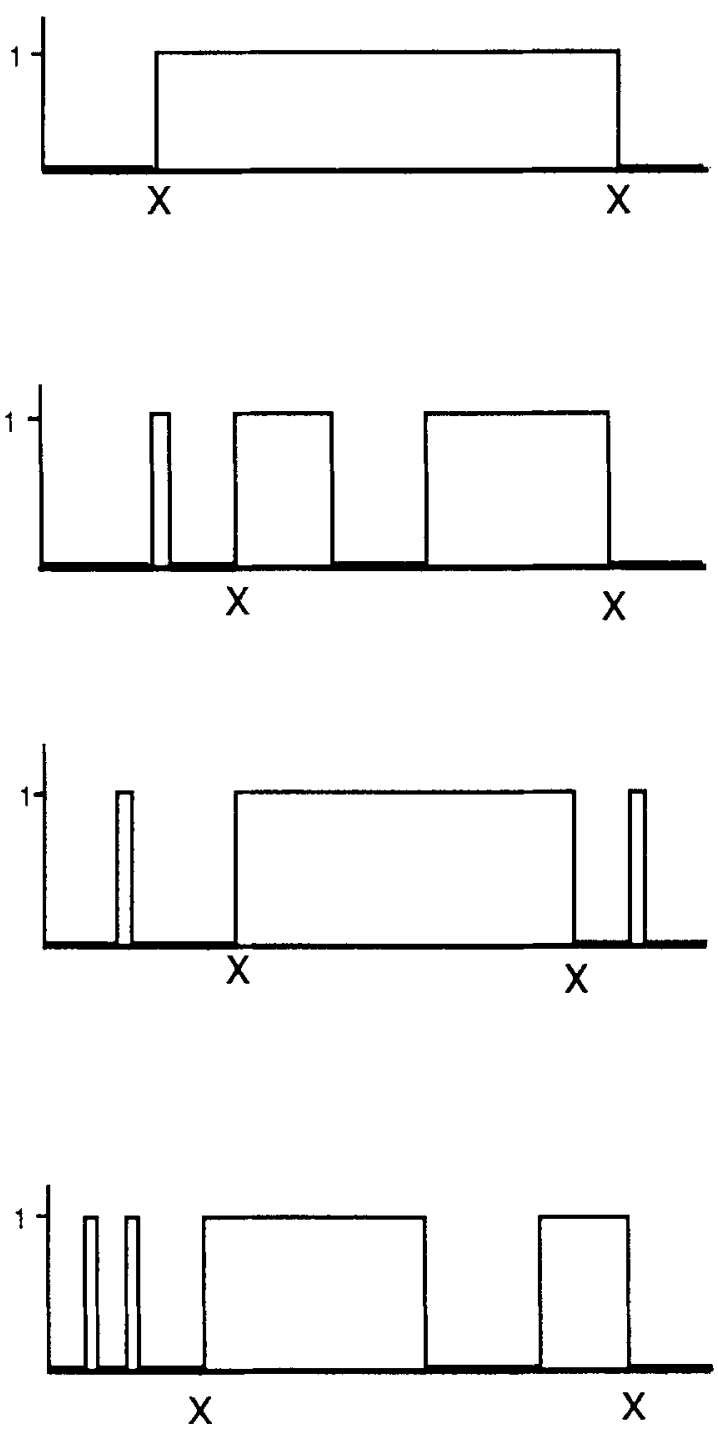

Fig 3. Examples of profiles, scanned within a thresholded image, that are used for finding the patient contour. The long, high-level plateau corresponds to the patient contour. The low-level bars inside the contour $\mathbf{x - x}$ reflect the dark lung area (below the thresholding value). The low-level bars outside the contour $x-x$ mark the presence of intraoperative tubes or noisy objects.

will be shifted, allowing the patient contour to be determined.

The distance between two points marked within a profile is defined as a profile length. On the basis of the minimum/maximum profilelength ratio, a PA (or AP) view is distinguished from a lateral projection. In the PA case, the maximum profile length shows the location of the upper extremities, whereas for the lateral projection, the minimum profile length marks the neck location. The orientation of the PA- view image is determined on the basis of the spine, abdomen and upper extremities. For lateral projections, only the spine and neck locations are used, eliminating two of four orientations of the image.

In the final step, the decision has to be made whether the orientation of the image is correct or needs a $y$-axis flip. The original image is thresholded once again. This time the threshold value is an average of all pixel values within the contour of the patient. After thresholding the entire image (Fig 4), the lung regions are extracted. By comparing the areas of the left and right lung regions, it can be determined whether the image needs to be flipped.

By combining the results of all three steps, the angle of the current orientation can be determined, and the image is rotated by one of seven rotation routines or is left in the original position.

\section{RESULTS AND DISCUSSION}

This report describes an algorithm that identifies the current image orientation with respect to the standard position viewed by a radiologist. A three-step procedure finds the mediastinum, locates the upper extremities, and compares the areas of the right and left lung regions. Based

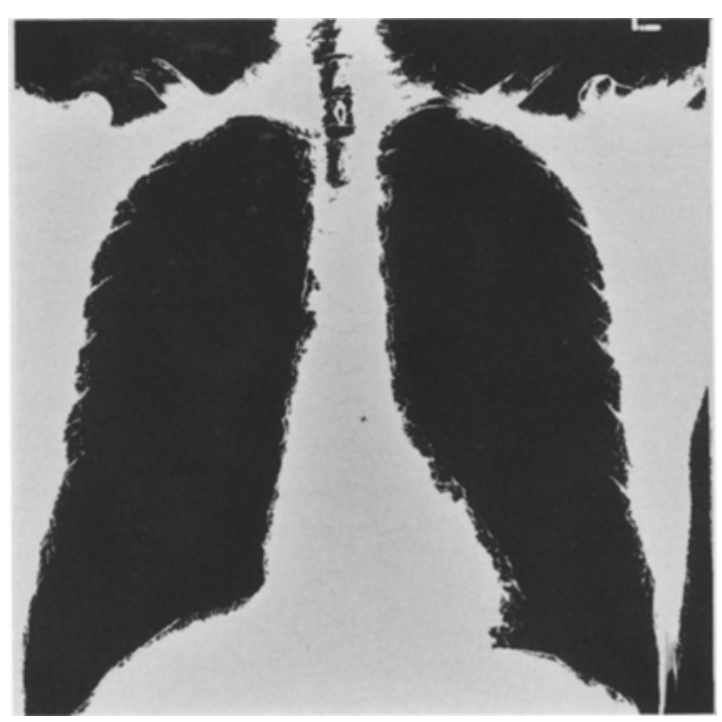

Fig 4. Thresholded chest image with extracted lung regions. 
on this analysis, one of eight angles is chosen to rotate the image.

This algorithm is used for PA (or AP) and lateral projections of adult and pediatric radiographs. The first two steps have been tested in the clinical environment for more than 6 months. The last step has recently been developed. The procedure has been implemented with a clustercontroller computer. Before an image is archived and transmitted to a workstation, it is reformatted and oriented to a standard position, allowing a standard data format for archiving and a standard image orientation for viewing on the display workstation.

The statistical analysis of 976 images within a 1-month period has been processed by the orientation procedure. The results (Table 1) show that of 976 images, only 45 chest images
Table 1. Results of the Orientation Procedure

\begin{tabular}{lcc}
\hline & $\begin{array}{c}\text { Rotated } \\
\text { Correctly }\end{array}$ & $\begin{array}{c}\text { Rotated } \\
\text { Incorrectly }\end{array}$ \\
\hline $\begin{array}{l}\text { Correct orientation } \\
\text { Incorrect orientation }\end{array}$ & $584(59.8 \%)$ & $17(1.8 \%)$ \\
\hline
\end{tabular}

(4.6\%) during a 1-month period had to be rotated manually instead of the 375 images $(38.4 \%)$ that would have had to be rotated if the procedure had been implemented. This significantly reduced the time that had to be spent by radiologists or technologists to rotate all images manually.

\section{ACKNOWLEDGMENT}

The authors thank Sandra Eldredge and Jim Tagawa for their assistance in implementing this procedure in the clinics and for providing feedback on its performance.

\section{REFERENCES}

1. Taira R, Huang HK: A picture archiving and communication system module for radiology. Comput Methods Programs Biomed 30:229-247, 1989

2. Huang HK: Image storage, transmission, and manipulation. J Minimally Invasive Ther 1:85-92, 1991

3. Huang HK, Taira R: PACS infrastructure design. AJR Am J Roentgenol 158:743-749, 1992
4. McNitt-Gray M, Pietka E, Huang HK: Image processing for PACS. Invest Radiol (in press)

5. Pietka E, McNitt-Gray M, Kuo ML, et al: Computer assisted phalangeal analysis in skeletal age assessment. IEEE Trans Med Imaging 10:616-620, 1991

6. McNitt-Gray M, Eldredge S, Tagawa J, et al: Automatic removal of unexposed background in digital radiographs. Proc SPIE 1653:94-102, 1992 